\title{
AMCoR
}

Asahikawa Medical University Repository http://amcor.asahikawa-med.ac.jp/

Gut (2011) 60(4):441.

An unusual elevated lesion of the oesophagus

Sawada, Koji ; Ikuta, Katsuya ; Itabashi, Kentaro ; Suzuki, Yasuyuki ; Mizukami, Yusuke ; Fujiya, Mikihiro ; Kubo, Koji ; Tamura, Yasuaki ; Torimoto, Yoshihiro ; Kohgo, Yutaka 


\section{An unusual elevated lesion of the oesophagus}

Koji Sawada ${ }^{1}$, Katsuya Ikuta ${ }^{2}$, Kentaro Itabashi $^{1}$, Yasuyuki Suzuki ${ }^{1}$, Yusuke Mizukami $^{2}$, Mikihiro Fujiya ${ }^{2}$, Koji Kubo ${ }^{1}$, Yasuaki Tamura ${ }^{3}$, Yoshihiro Torimoto $^{2}$, Yutaka Kohgo ${ }^{2}$

${ }^{1}$ Department of Internal Medicine, Municipal Nakashibetsu Hospital, Nakashibetsu; ${ }^{2}$

Department of Medicine, Division of Gastroenterology and Hematology/Oncology, Asahikawa Medical College, Asahikawa; ${ }^{3}$ Department of Pathology, Sapporo Medical University School of Medicine, Sapporo, Japan.

Corresponding author:

Katsuya Ikuta, M.D., Ph.D.

Assistant Professor

Division of Gastroenterology and Hematology/Oncology, Department of Medicine Asahikawa Medical College 2-1-1-1 Midorigaoka-Higashi Asahikawa, Hokkaido 078-8510, Japan 078-8510

TEL: +81-166-68-2462

FAX: +81-166-68-2469

e-mail: ikuta@asahikawa-med.ac.jp 


\section{CLINICAL PRESENTATION}

A 44-year-old man with a history of gastric cancer that was treated with distal gastrectomy 4 years ago underwent upper endoscopic examination in a follow-up study. The endoscopic examination revealed an elevated lesion $8 \mathrm{~mm}$ in size in the lower esophagus (Figure 1A) and narrow band imaging (NBI) endoscopy showed vascular augmentation as brownish spots on the surface of the elevated lesion (Figure 1B). There was no abnormality in the stomach and esophagus-squamous junction. Physical examination and a routine blood test, including tumor markers, showed no abnormal findings. Whole body computed tomography (CT) detected no abnormalities. A biopsy of the lesion was performed.

\section{Figure legends}

\section{Figure 1.}

(A) Endoscopic photograph of the lower esophagus showing an elevated lesion. (B) Narrow band imaging (NBI) endoscopy displaying brownish spots on the surface of the lesion. 


\section{QUESTION}

What is the diagnosis?

\section{ANSWER}

Histological findings showed that massive centrocyte-like (CCL) cells were diffusely infiltrated in the lamina propria (Figure 2A, B). CCL cells destroyed the esophageal grand and formed lymphoepithelial lesion (LEL). The atypical cells were detected $\mathrm{CD}_{20}^{+}, \mathrm{CD} 79 \mathrm{a}^{+}, \mathrm{CD}^{-}$and $\mathrm{CD}^{-} 0^{-}$, corroborating the diagnosis of a primary esophageal mucosa-associated lymphoid tissue (MALT) lymphoma. Administration of $60 \mathrm{mg}$ lansoprazole, $1500 \mathrm{mg}$ amoxicillin and $800 \mathrm{mg}$ clarithromycin for two weeks was undertaken, similar to eradication therapy for gastric MALT lymphoma. Endoscopic and pathological examination performed on 6 months after treatment detected no evidence of lymphoma (Figure 2C, D).

Primary esophageal MALT lymphoma has been reported less frequently and the best therapeutic strategy has not yet been established [1-5] (Table). 
This is the first case of esophageal MALT lymphoma cured with antibiotics and proton pump inhibitor (PPI). Our case has shown no recurrence for one year, in support of administration of antibiotics and PPI as a new option for managing esophageal MALT lymphoma.

\section{Figure legends}

\section{Figure 2.}

(A) Massive atypical lymphoid cells were noted in the esophageal lamina propria (hematoxylin-eosin, x100). (B) CCL cells destroyed the esophageal grand and formed lymphoepithelial lesion (LEL) (hematoxylin-eosin, x200). (C) Endoscopic photograph 6 months after administration of antibiotics and proton pump inhibitor detected no lymphoma lesion. (D) Histological findings of biopsy specimens taken from lower esophagus showed no lymphoma cells (hematoxylin-eosin, x200). 


\section{REFERENCES}

1 Nishiyama Y, Yamamoto Y, Ono Y, et al. Visualization of esophageal non-Hodgkin’s lymphoma with Ga-67 scintigraphy. Ann Nucl Med 1999;13:419-421.

2 Hosaka S, Nakamura N, Akamatsu T, et al. A case of low grade mucosa associated lymphoid tissue (MALT) lymphoma of the oesophagus. Gut 2002;51: 281-284.

3 Kitamoto Y, Hasegawa M, Ishikawa H, et al. Mucosa-associated lymphoid tissue lymphoma of the esophagus: a case report. J Clin Gastroenterol 2003;36:414-416.

4 Shim CS, Lee JS, Kim JO, et al. A case of primary esophageal B-cell lymphoma of MALT type, presenting as a submucosal tumor. J Korean Med Sci 2003;18:120-124.

5 Miyazaki T, Kato H, Masuda N, et al. Mucosa-associated lymphoid tissue lymphoma of the esophagus : case report and review of the literature. Hepato-Gastroenterology 2004;51:750-753. 
Table 1: Clinical and pathological findings of primary esophageal MALT lymphoma

\begin{tabular}{|c|c|c|c|c|c|c|}
\hline Author & Age & Sex & Appearance & Therapy & Clinical course & Location \\
\hline 1 Nishiyama et al ${ }^{1}$ & 63 & $\mathrm{~F}$ & SMT $10 \mathrm{~cm}$ & NA & NA & $25 \mathrm{~cm}^{*}$ \\
\hline 2 Hosaka et $\mathrm{al}^{2}$ & 83 & $\mathrm{~F}$ & two SMTs $1 \mathrm{~cm}$ & EMR & 22 months & 20 cm* \\
\hline 3 Kitamoto et al ${ }^{3}$ & 74 & $\mathrm{~F}$ & SMT & Radiation & NA & 29 cm* \\
\hline 4 Shim et al ${ }^{4}$ & 61 & M & SMT $8 \mathrm{~cm}$ & Surgery & NA & mid to distal \\
\hline 5 Miyazaki et al ${ }^{5}$ & 49 & M & $\mathrm{SMT} 4 \mathrm{~cm}$ & Surgery & NA & $36 \mathrm{~cm} *$ \\
\hline 6 Our case & 44 & M & Elevation $8 \mathrm{~mm}$ & $\begin{array}{l}\text { Administration of } \\
\text { antibiotics and PPI }\end{array}$ & One year & Distal \\
\hline
\end{tabular}

SMT: submucosal tumor; EMR: endoscopic mucosal resection; PPI: proton pump inhibitor; NA: not available; *: distance from incisor

teeth 


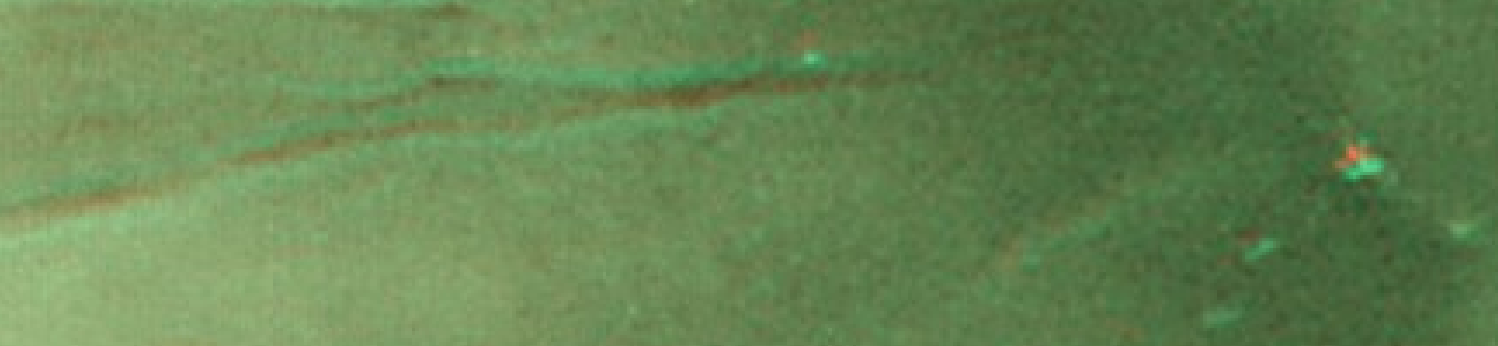

is

.
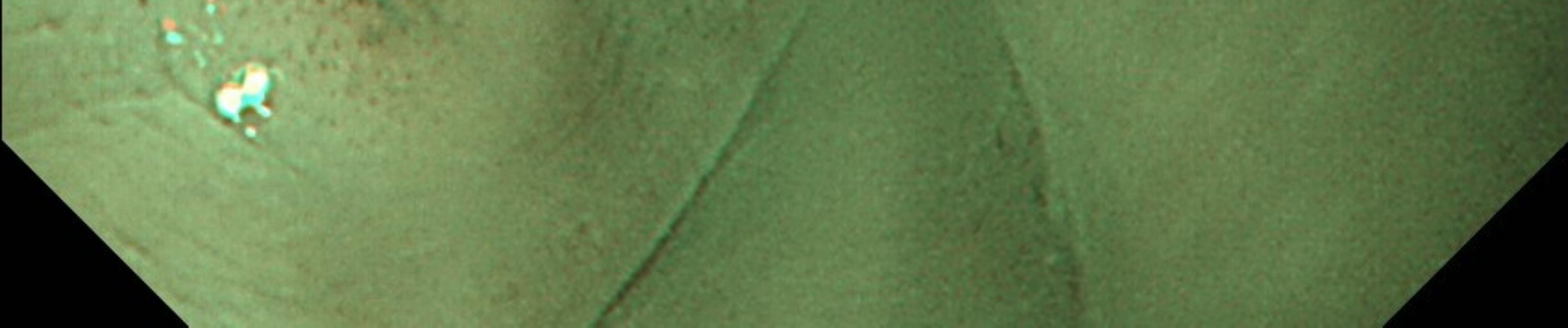
313 of

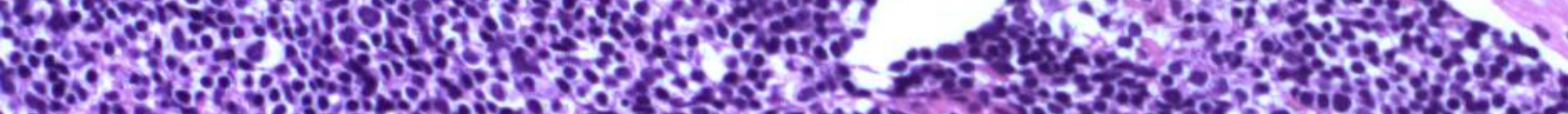

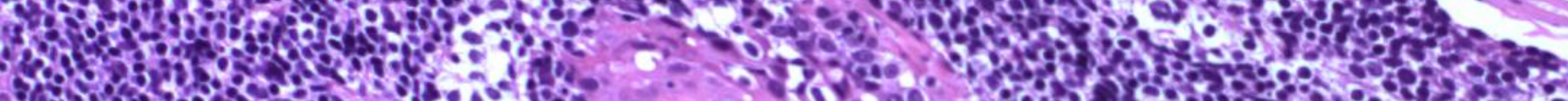

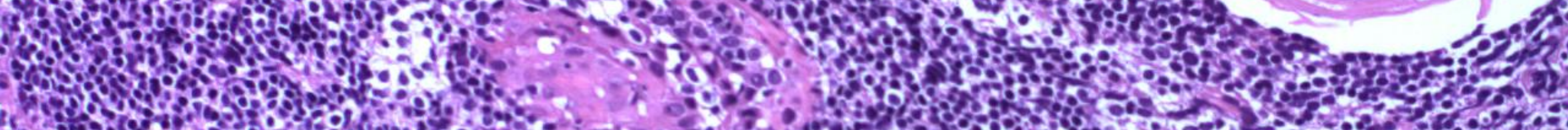

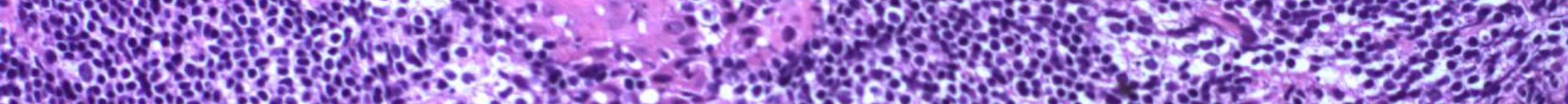
AH

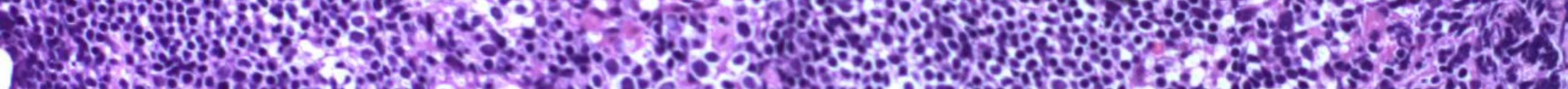

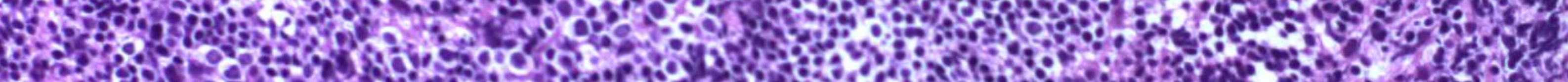

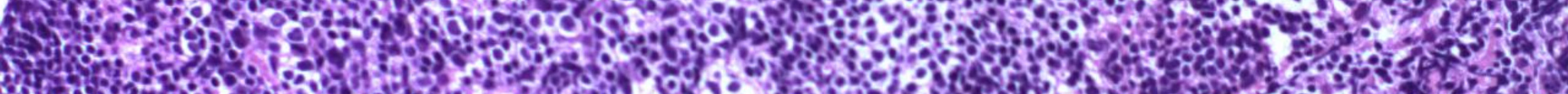

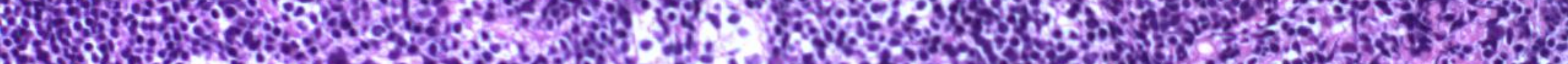

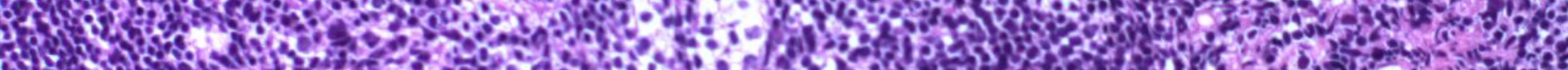

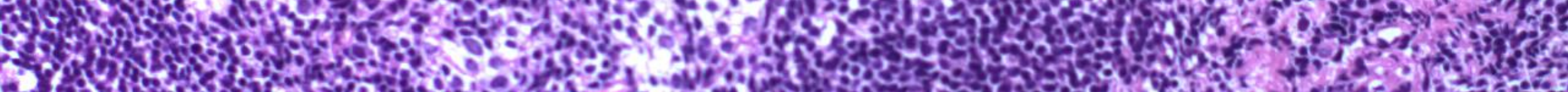

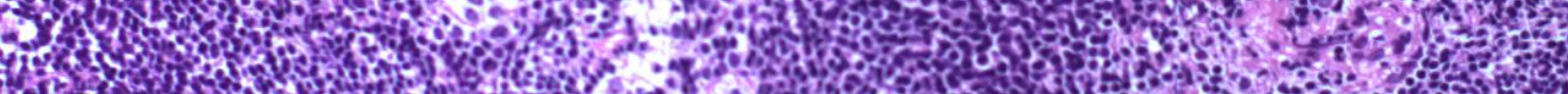

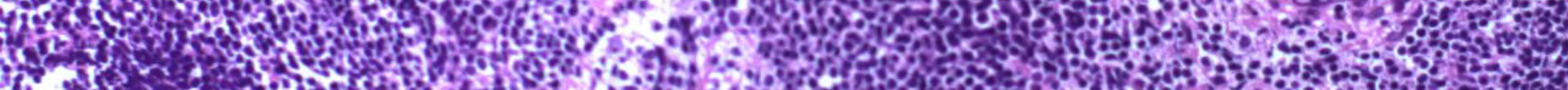
H.

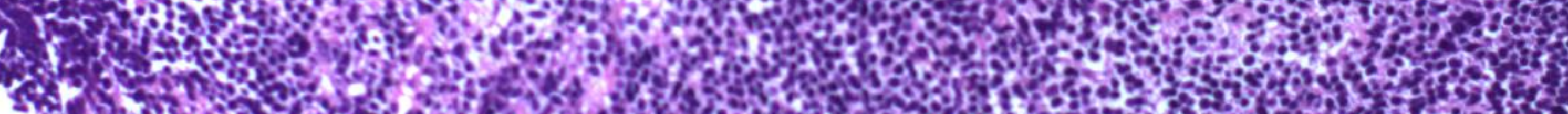

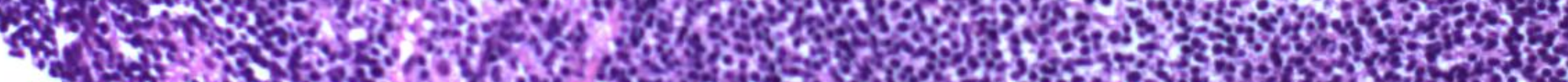

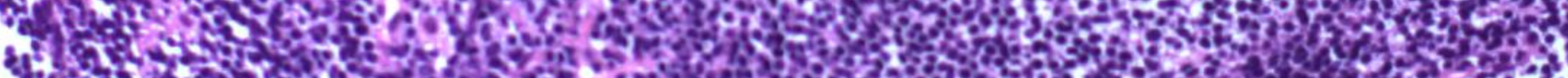
6.

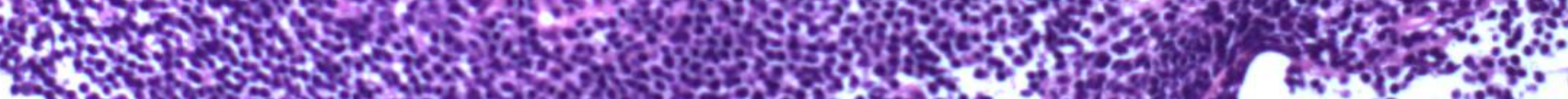
1.5. 13. 3 .

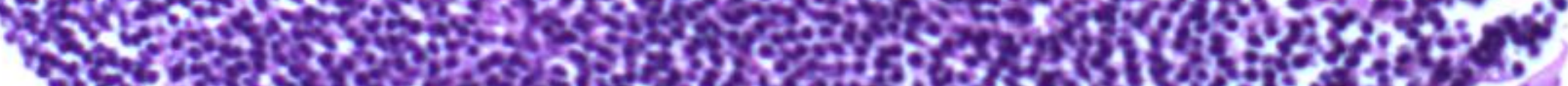

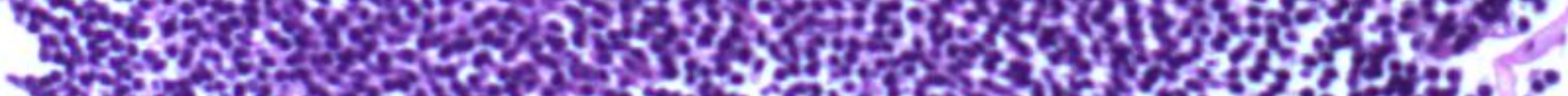

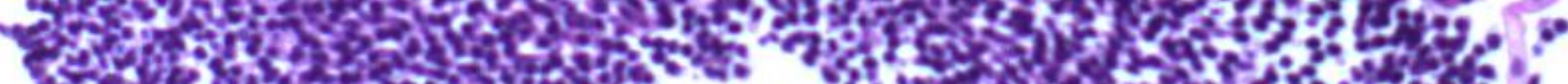

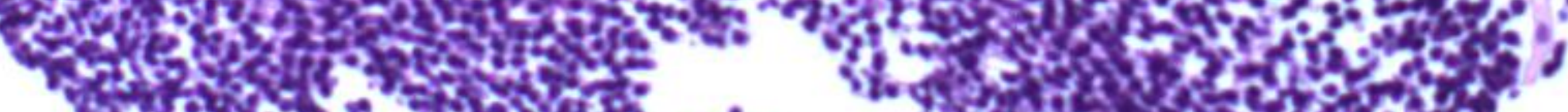

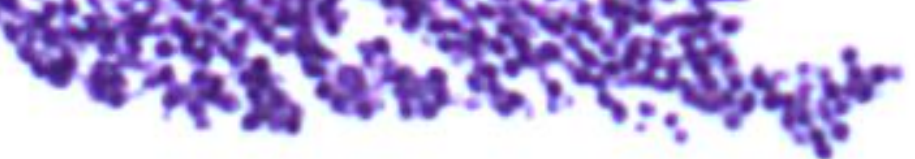

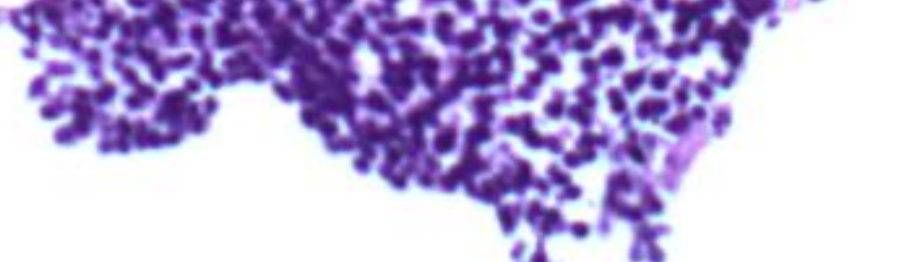




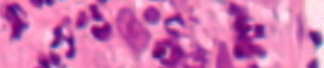

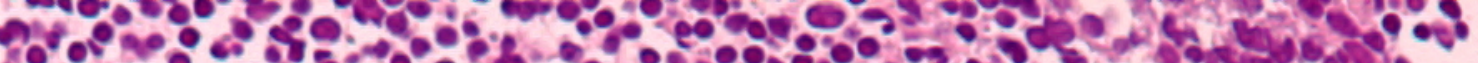

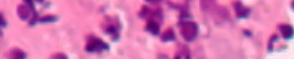

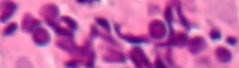

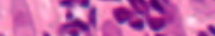

(i) $190^{\circ}$

5 and

(4) 0.

18

40

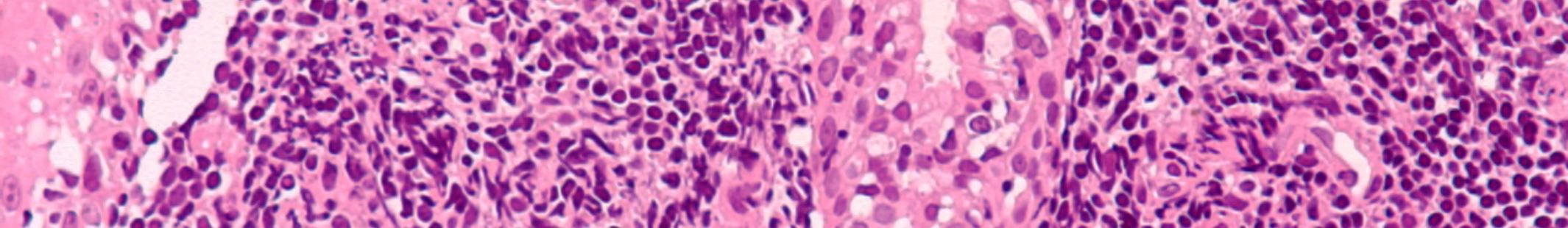

- 0 ry

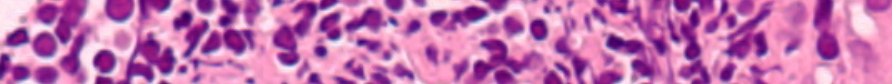

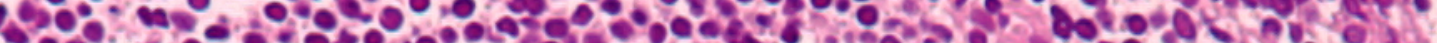

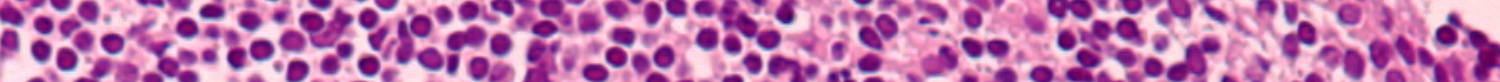

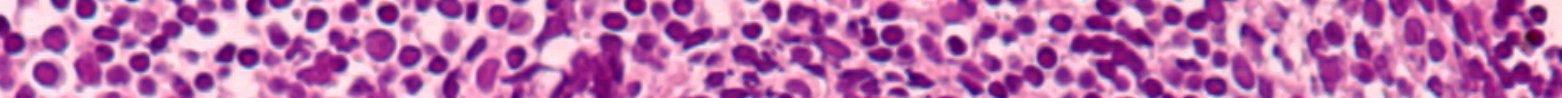

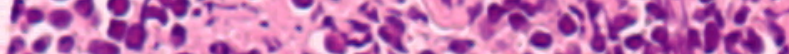

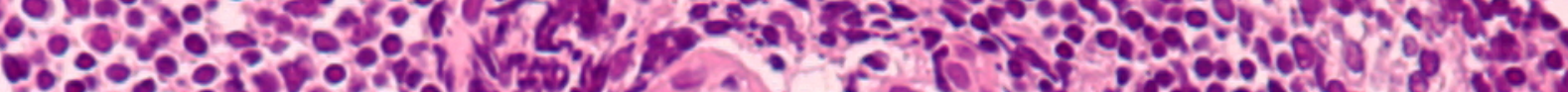

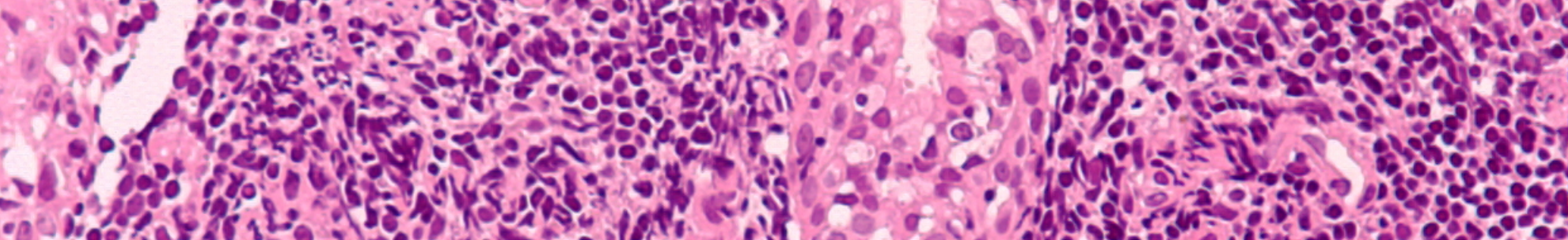

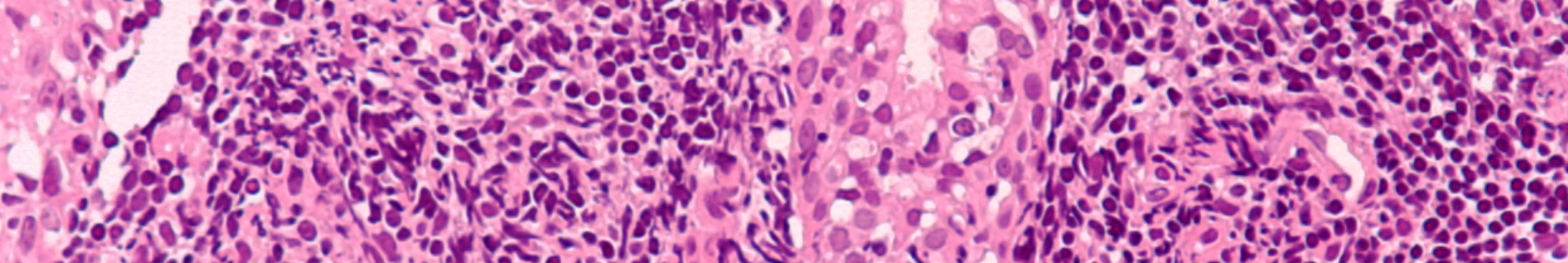

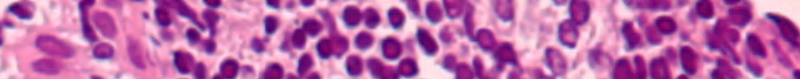

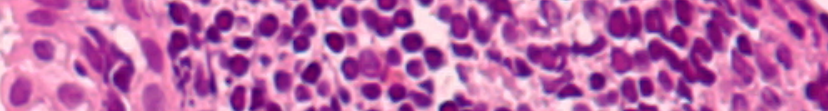

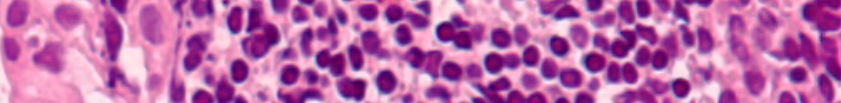

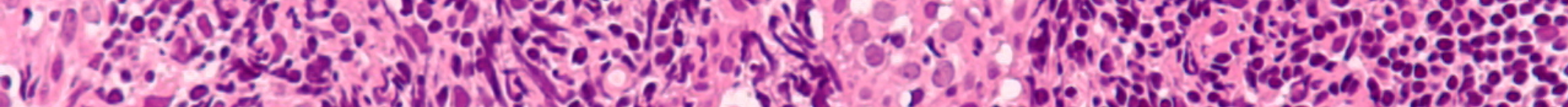

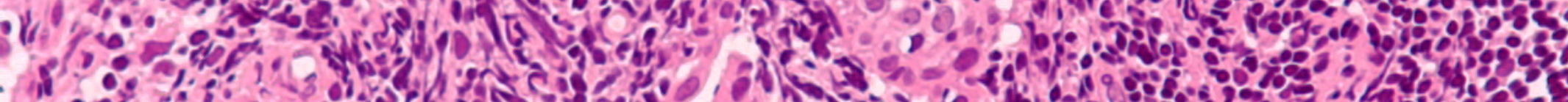

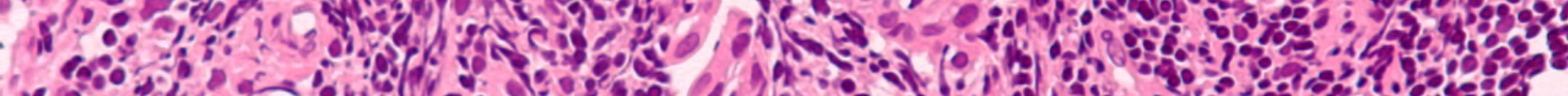

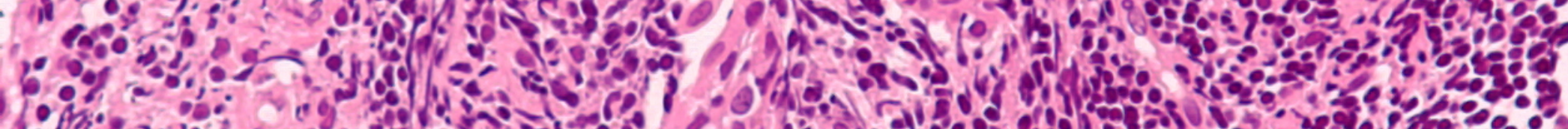

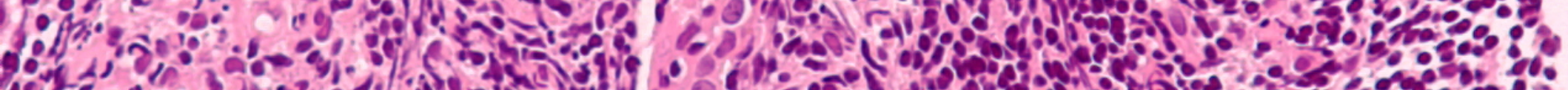

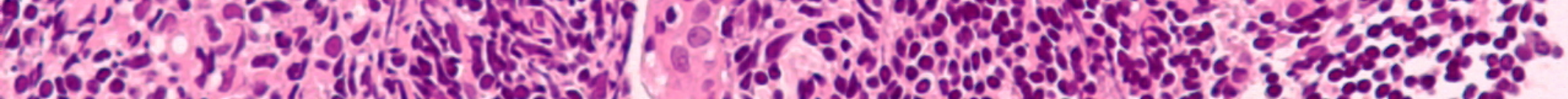
elos

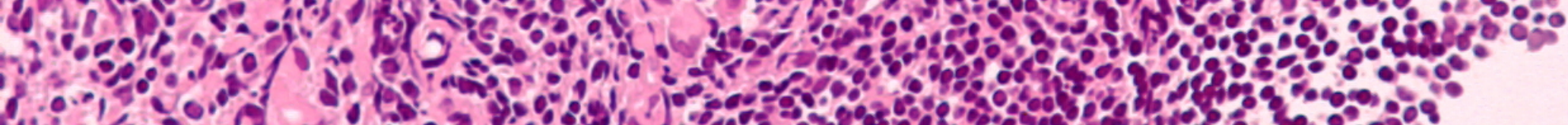
fi.

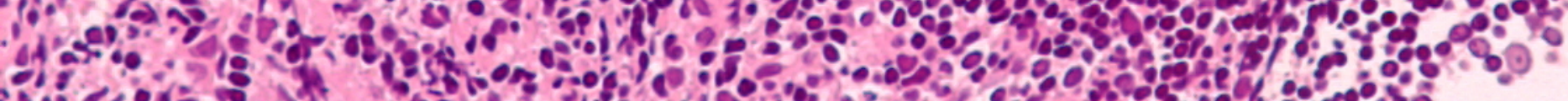

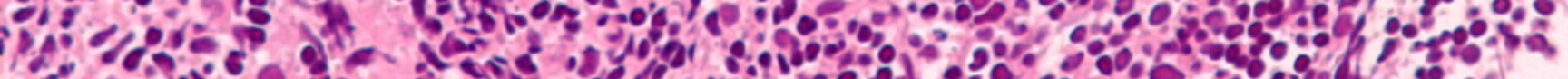
31

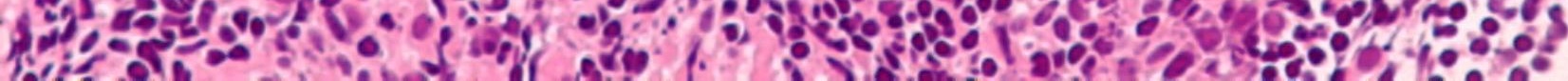



$a_{0}^{-1}=b^{2}=$

(a) $x^{2}+3$<smiles>CCCC1CCCCC1</smiles>

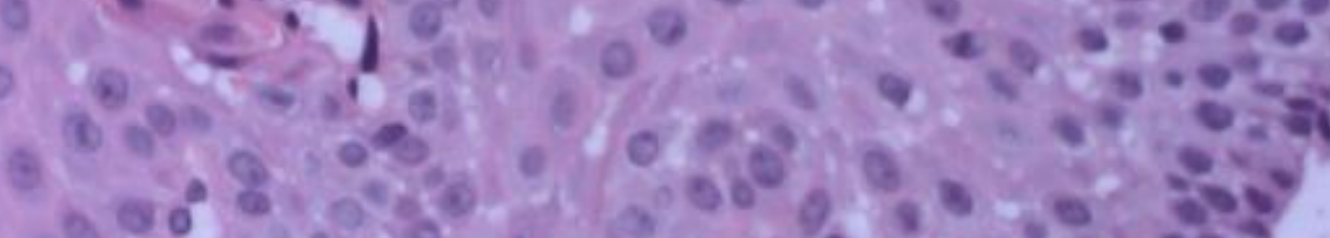

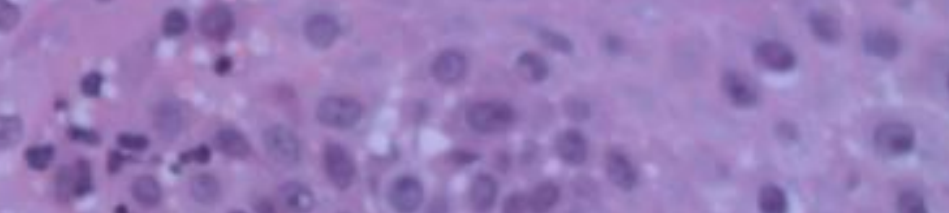

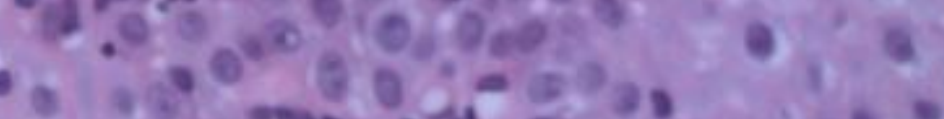

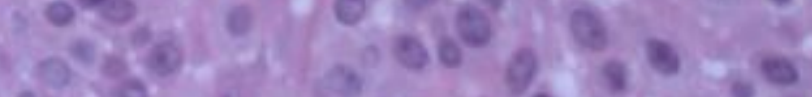

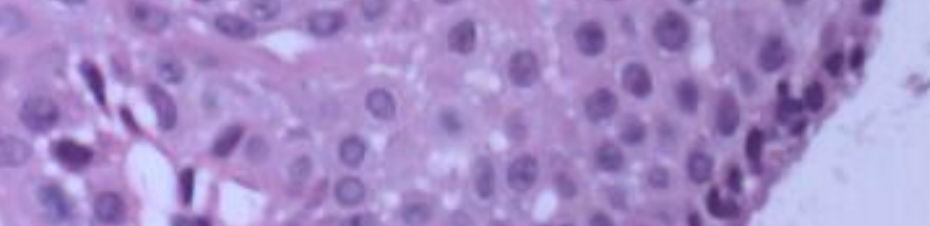
B. $00.0 \%$.

Q8. 20.0.

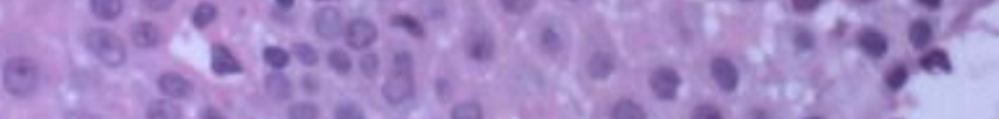

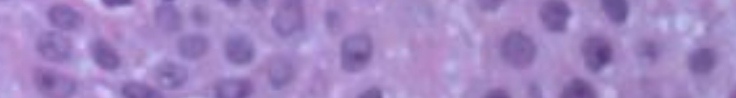

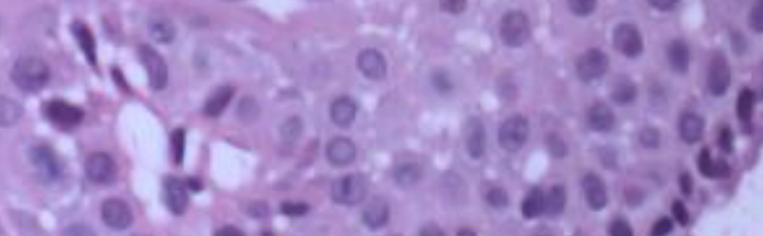

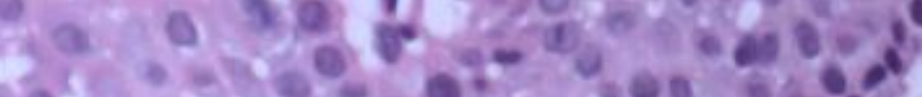

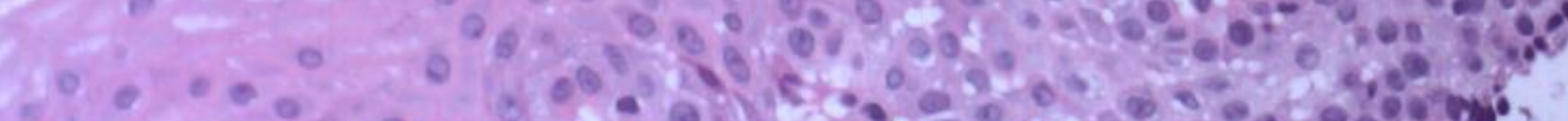

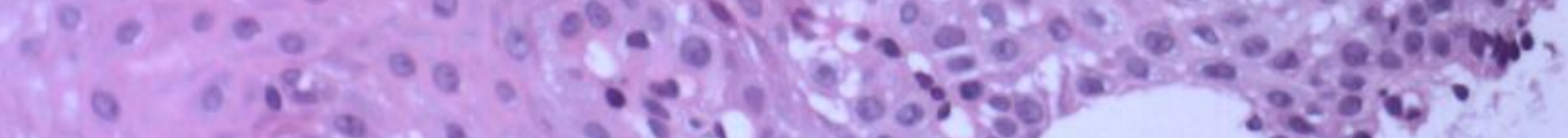

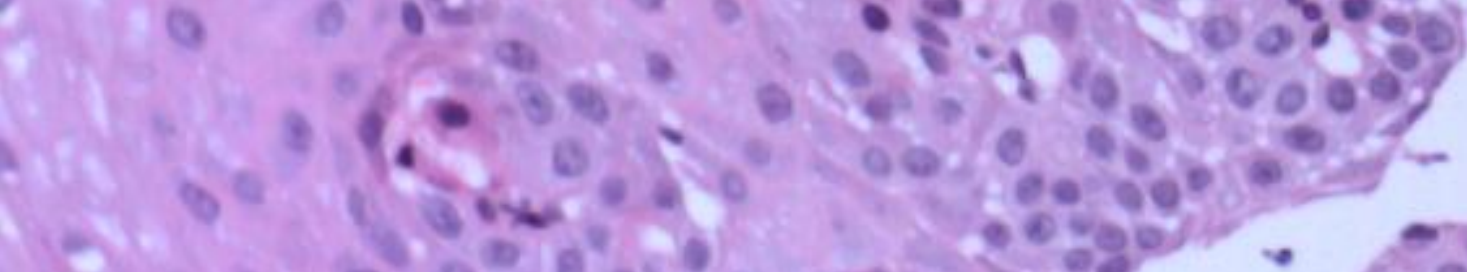

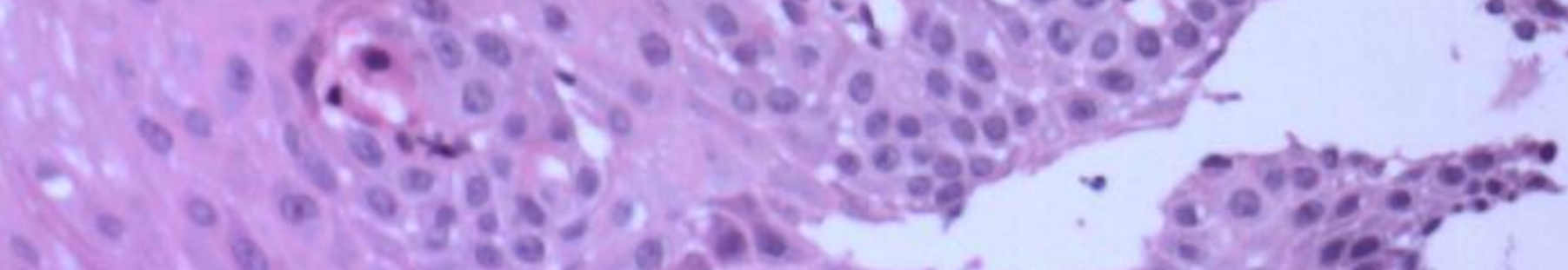

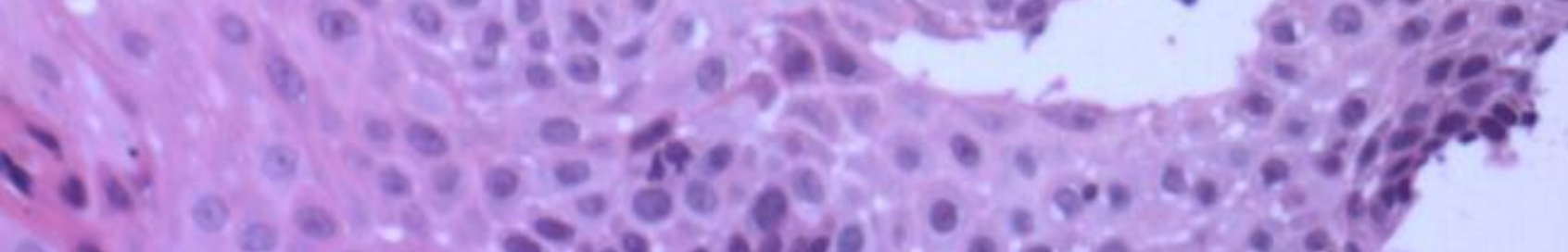

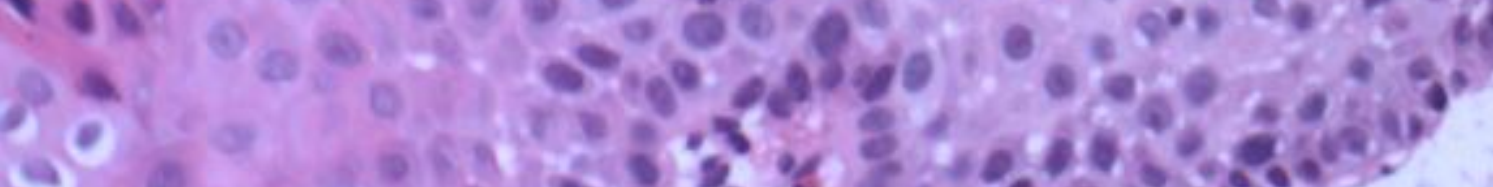

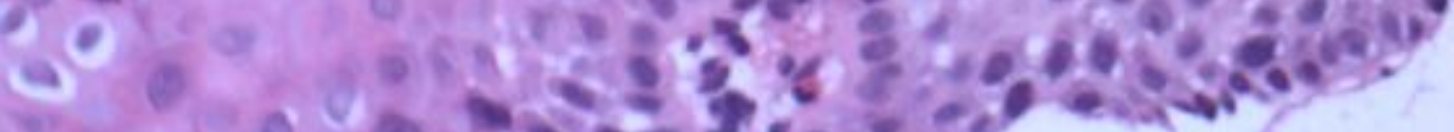
ra
30,503 a

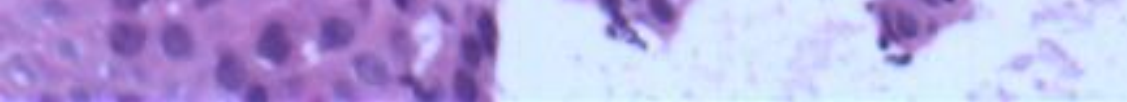

